\title{
Processo de cuidar do idoso em Diálise Peritoneal Ambulatorial Contínua no domicílio*
}

\author{
Home care for the elderly undergoing Continuous Ambulatory Peritoneal Dialysis
}

\author{
Proceso de cuidar del anciano, que hace Diálisis Peritoneal Ambulatorial Contínua en el \\ domicilio
}

\author{
Daniele Favaro Ribeiro', Sueli Marques ${ }^{2}$, Luciana Kusumota ${ }^{2}$, \\ Rita de Cássia Helu Mendonça Ribeiro ${ }^{3}$
}

\section{RESUMO}

Objetivo: Caracterizar os idosos com insuficência renal crônica termina em tratamento de diálise peritoneal ambulatorial contínua e seus cuidadores e descrever o processo de cuidadr desses idosos. Métodos: Estudo de abordagem qualitativa com dados coletados por meio de entrevista com nove cuidadores utilizando a história oral temática e a análise temática dso dados. Resultados: Dentre os nove idosos, cinco eram homens, média de idade 70 anos e todos dependiam do cuidador para troca da bolsa de diálise. Dos cuidadores, oito eram mulheres, média de idade 41,5 anos e despencia oito horas diárias para o cuidado. As falas referentes à categoria processo de cuidar do idoso com IRCT em DPACl contínua no domicílio. Conclusão: O estudo revelou a necessidade de auxiliar o cuidador a desenvolver conhecimentos e habilidades para lidar com a demanda de cuidados que o idoso exige, principalmente, em relação a DPAC.

Descritores: Insuficiência renal crônica; Diálise peritoneal; Cuidadores; Idoso.

\begin{abstract}
Objectives: To describe the elders with end stage renal disease (ESRD) undergoing continuous ambulatory peritoneal dialysis (CAPD), their caregivers, and the care the caregivers provide to the elders. Methods: This was a qualitative study with 9 caregivers. Data were collected through oral history. Data analysis consisted of thematic content analysis. Results: The sample consisted of 5 male and 4 female elders and all them were dependent on caregivers to change the dialysis collection bag. The mean age of the participants was 70 years. Among the caregivers, 8 of them were female with a mean age of 41.5 years and they provided 8 hours of care to the elders daily. The main theme emerging from the content analysis was "home care for the elderly undergoing continuous ambulatory peritoneal dialysis." Conclusion: Caregivers need support for the development of knowledge and skills to deal with the elders' demand of care, particularly in regard to the management of CAPD.
\end{abstract}

Keywords: Renal insufficiency, chronic; Peritoneal dialysis; Caregivers; Aged.

\section{RESUMEN}

Objetivos: Caracterizar a los ancianos con insuficiencia renal crónica terminal (IRTC) en tratamiento de diálisis peritoneal en ambulatorio y la continuación (DPAC) del cuidado en el domicilio; caracterizar a sus cuidadores; y, describir el proceso de cuidar de esos ancianos. Métodos: Se trata de un estudio con abordaje cualitativo, utilizando la historia oral temática para la recolección de datos con nueve cuidadores y el análisis temático de los datos. Resultados: Entre los nueve ancianos, cinco eran hombres; promedio de edad 70 años; todos dependían del cuidador para cambiar la bolsa de diálisis. Entre los cuidadores, ocho eran mujeres; promedio de edad 41,5 años y dedicaban ocho horas diarias al cuidado. En el análisis, fue constituida la categoría: el proceso de cuidar del anciano con IRCT en DPAC en el domicilio. Conclusión: El estudio reveló la necesidad de auxiliar al cuidador a desarrollar conocimientos y habilidades para lidiar con la demanda de cuidados que el anciano exige, principalmente, en relación a DPAC.

Descriptores: Insuficiencia renal crónica; Diálisis peritoneal; Cuidadores; Anciano.

\footnotetext{
* Trabalho realizado na Escola de Enfermagem de Ribeirão Preto da Universidade de São Paulo - USP - Ribeirão Preto (SP), Brasil. ${ }^{1}$ Pós-graduanda (Mestrado) da Escola de Enfermagem de Ribeirão Preto da Universidade de São Paulo - USP - Ribeirão Preto (SP), Brasil.

${ }^{2}$ Professora Doutora do Departamento de Enfermagem Geral e Especializada da Escola de Enfermagem de Ribeirão Preto da Universidade de São Paulo - Ribeirão Preto (SP), Brasil.

${ }^{3}$ Pós-graduanda (Doutorado). Professora da Faculdade de Medicina de São José do Rio Preto- FAMERP. São José do Rio Preto (SP), Brasil.
} 


\section{INTRODUÇÃO}

O fenômeno envelhecimento é tema de atenção no âmbito mundial. No Brasil, este fenômeno vem acontecendo devido a mudanças no perfil demográfico da população, na qual a faixa etária de 60 anos e mais de idade é a que mais cresce em termos proporcionais. A partir de 1940, a mortalidade começou a declinar no país, assim como a taxa de fecundidade, que caiu de seis para dois filhos por mulher. Com a diminuição do número de nascimentos, a proporção de todas as outras faixas etárias aumentou, elevando a média de idade da população ${ }^{(1-2)}$.

Dessa forma com o envelhecimento populacional ocorreram alterações no perfil de morbimortalidade, no qual se observa a substituição das doenças infecto-contagiosas pelas crônicas não transmissíveis, que podem trazer complicações, incapacidades e, conseqüentemente, a diminuição da qualidade de vida dos idosos ${ }^{(1)}$.

As doenças crônicas acometem, na maioria das vezes, pessoas com idade mais avançada. Este resultado constitui motivo de preocupação para os profissionais de saúde, devido aos seguintes aspectos: o tratamento de saúde, no qual ocorre o desgaste e sofrimento da pessoa doente e da família, com comprometimento da situação econômica; e o fato de que os recursos financeiros e humanos dos serviços públicos priorizam atividades curativas e de reabilitação, ao invés de ações preventivas e de promoção da saúde ${ }^{(3)}$.

Dentre as doenças crônicas não transmissíveis, encontrase a insuficiência renal crônica terminal (IRCT), que se refere a um diagnóstico sindrômico de perda progressiva e, geralmente, irreversível da função renal de depuração, ou seja, da filtração glomerular ${ }^{(4)}$. A incidência dessa doença vem crescendo em virtude do aumento da longevidade da população e, principalmente, por complicações de doenças sistêmicas, como a hipertensão arterial e o diabetes mellitus ${ }^{(5)}$.

A diálise peritoneal ambulatorial contínua (DPAC), uma modalidade de tratamento da IRCT, é um procedimento que pode ser realizado no domicílio e consiste na realização de trocas das bolsas de diálise, que o próprio cliente ou cuidador devidamente treinados podem realizar. O líquido dialítico é infundido por meio de um cateter na cavidade abdominal, permanecendo por seis a oito horas, onde ocorre a osmose e difusão de solutos através da membrana peritoneal. Após o tempo de permanência do líquido no abdome, este é drenado e substituído por uma nova solução ${ }^{(6-7)}$.

$\mathrm{O}$ idoso com IRCT que apresenta incapacidade para realizar sozinho o procedimento de DPAC necessita de auxílio. A pessoa com potencial para assumir esta responsabilidade passa por um processo de ensinoaprendizagem, planejado e realizado pelo enfermeiro, no qual adquire conhecimentos específicos sobre a técnica e como identificar as possíveis intercorrências e complicações relacionadas ao tratamento. Assume atividades como, acompanhamento nos retornos mensais ou quinzenais, cuidados com o cateter, administração de medicamentos de uso diário e contínuo, realização da técnica de DPAC, controle da alimentação e da ingesta de líquidos, supervisão das atividades cotidianas do idoso, entre outras ${ }^{(6)}$.

O tratamento de DPAC, porém, possuí dificuldades, pois o cuidador e o idoso devem ter sempre em mente os princípios de assepsia, higiene e limpeza para evitar a peritonite, e necessitam de atenção para o manejo do cateter. Na prática, o cotidiano do cliente em DPAC modifica-se, pois possuí restrições físicas e sociais o que impede a participação em atividades. Sobretudo, para o cuidador, sua atividade com esse tratamento impossibilita sua inserção no mercado de trabalho, levando ao comprometimento do orçamento familiar e à dependência financeira de outros ${ }^{(8-9)}$.

Estudos demonstram que devido à sobrecarga de cuidados, os cuidadores, podem apresentar desespero, isolamento, fadiga intensa, redução do tempo livre e do envolvimento com a comunidade e atividades religiosas. Acrescentam que os sintomas de fadiga são complexos, podem estar associados com a depressão e, muitas vezes, não são diagnosticados ${ }^{(10,11-12)}$.

Em muitos casos, a função de cuidador é assumida por uma única pessoa, o cuidador principal, ou seja, o que assume tarefas de cuidado, atendendo às necessidades do idoso e responsabilizando-se por elas. Como o cuidado dos pacientes em DPAC são realizados no domicílio, com freqüência a cuidadora primária é a esposa, seguida da filha. E, o ato de cuidar gera sentimentos diversos e contraditórios, medo, angústia, cansaço, tristeza e choro ${ }^{(12)}$.

Considerando-se a relevância dessa temática e a atuação como enfermeira assistencial e na docência no setor de nefrologia, e especificamente no tratamento da DPAC, evidenciou-se a importância do cuidador no tratamento de pessoas com IRCT, principalmente os idosos. Dessa forma, questionou-se: como é o processo de cuidar do idoso com IRCT em DPAC no domicílio?

Assim, o presente estudo objetivou caracterizar os idosos e seus respectivos cuidadores quanto aos dados sóciodemográficos, condição de saúde-doença, desempenho das atividades básicas e instrumentais da vida diária dos idosos e cuidados prestados pelo cuidador, bem como descrever o processo de cuidar de idosos com IRCT em DPAC atendidos na unidade de diálise de um hospital escola do interior paulista, com o intuito de subsidiar o planejamento da assistência de enfermagem, tanto para o idoso quanto para o cuidador.

\section{MÉTODOS}

Trata-se de uma pesquisa de abordagem qualitativa, na qual os dados foram coletados por meio de entrevista, utilizando a história oral temática ${ }^{(13)}$ é constituída pela reconstrução histórica de vida do sujeito, a partir de um dado momento ou evento que se deseja compreender, neste caso, o cuidado do idoso com IRCT em tratamento DPAC no domicílio.

A história oral temática realiza-se a partir de um assunto específico e pré-estabelecido, no qual há o comprometimento com o esclarecimento ou opinião do entrevistador sobre 
algum evento definido. Neste gênero, a participação do entrevistador é mais evidente. A objetividade é direta. Não só admite o uso do questionário, mas também se torna peça fundamental para aquisição dos detalhes procurados ${ }^{(13)}$.

Este estudo foi realizado com nove cuidadores de idosos com diagnóstico clínico de IRCT, em tratamento de DPAC, inseridos no programa do Serviço de Nefrologia de um hospital escola do interior paulista, no período de agosto de 2007 a janeiro de 2008.

A princípio, os cuidadores foram contatados durante as consultas de enfermagem no hospital, onde foram orientados quanto aos objetivos do estudo e, posteriormente, as entrevistas foram agendadas previamente, por telefone, em dia e horário conforme disponibilidade do cuidador.

As entrevistas foram realizadas no domicílio do cuidador e se iniciaram após o consentimento individual, por escrito. Para tanto, foi utilizado um instrumento de coleta de dados desenvolvido ${ }^{(14)}$ e adaptado para este estudo, contendo dados de caracterização dos idosos e seus respectivos cuidadores, referentes ao perfil sócio-demográfico, condição de saúdedoença, desempenho para as atividades básicas e instrumentais da vida diária e as questões referentes aos cuidados prestados. Foram incluídas indagações referentes a IRCT e o tratamento de DPAC, além da questão orientadora: Fale-me como está sendo para você cuidar de um idoso com IRCT em DPAC. Cada entrevista durou, em média 65 minutos, as histórias foram registradas em fitas cassetes por meio de um gravador, e posteriormente transcritas, na integra, para possibilitar a análise. As histórias das experiências de cuidado passaram por um processo de transformação, do relato oral, para um texto escrito.

Para análise das histórias, adotou-se a análise temática que consiste em descobrir os núcleos de sentido que fazem parte de uma comunicação, nos quais a presença ou freqüência de aparição podem ter algum significado para o objeto analítico escolhido ${ }^{(15)}$. Esta análise divide-se em três fases: pré-análise, exploração do material e o tratamento dos resultados obtidos e interpretação, de onde emergiram aspectos do processo de cuidar de idosos com IRCT em DPAC, no domicílo.

O projeto de pesquisa foi aprovado pelo Comitê de Ética em Pesquisa da Faculdade de Medicina de São José do Rio Preto-SP (FAMERP), Parecer $\mathrm{n}^{\circ}$. 180/2007. Os idosos e cuidadores participantes da pesquisa assinaram os Termo de Consentimento Livre e Esclarecido.

\section{RESULTADOS}

Os nove idosos com IRTC em tratamento de DPAC que recebiam cuidados no domićlio, pelos cuidadores participantes deste estudo, eram procedentes da cidade de São José do Rio Preto e região.

A idade dos idosos variou entre 60 e 81 anos, com média de 70 anos; cinco do sexo masculino, oito casados, oito aposentados e quatro com ensino fundamental incompleto. Todos os idosos residiam em casa de alvenaria, com saneamento básico, sendo que a maioria residia em casa própria e adaptaram um dormitório para a realização do procedimento, colocando pia para facilitar a lavagem das mãos e retirando móveis em excesso.

O tempo em que estavam recebendo cuidados variou entre um e seis anos. Todos residiam em companhia de outras pessoas, seis moravam com seus respectivos cuidadores. Os idosos, em sua totalidade, não realizavam a troca das bolsas de diálise e recebiam ajuda de familiares para a realização das trocas e a manutenção da higiene do cômodo.

Quanto às condições de saúde-doença evidenciou-se, em relação à descoberta da IRC, que cinco descobriram a doença há um e quatro anos, três entre cinco e oito e três há mais de oito anos. De acordo com o relato dos cuidadores sobre os problemas de saúde apresentados pelos idosos, constatou-se que todos eram hipertensos, seis tinham problemas cardíacos, seis diabetes, cinco dificuldade visual, cinco problemas para dormir, quatro má circulação (varizes). Todos faziam uso de medicações por ordem médica, e a prática de automedicação foi negada.

Quanto aos nove cuidadores, a maioria era do sexo feminino, sete referiam ser casados, e com idade que variou entre 23 e 70 anos, com média de 41,5 anos. Em relação ao grau de parentesco com o idoso, três dos cuidadores eram filhas, cinco esposas, dois noras, uma neta. Quanto ao grau de escolaridade, duas tinham curso superior completo (fisioterapia e psicologia), porém não exerciam a profissão, duas completaram o ensino médio, duas tinham ensino fundamental completo e duas ensino fundamental incompleto.

No que se refere à profissão dos cuidadores, dois eram aposentados, duas manicures, duas do lar, um vendedor, uma técnica em enfermagem e uma estudante. Todos os cuidadores, além de prestarem cuidados aos idosos, também cuidavam da casa, estudavam, ou trabalhavam fora.

A renda familiar destes cuidadores variou de um a oito salários mínimos (SM) por mês, sendo que seis recebiam de dois a quatro SM, dois recebiam um SM; e um oito ou mais. É importante ressaltar que o salário mínimo vigente na época da coleta de dados era de $R \$ 380,00$ s.

Quando indagados sobre os problemas de saúde existentes entre os cuidadores, observou-se que quatro referiram não possuir nenhum problema de saúde, três referiram problemas para dormir, três problemas de coluna, dois pressão alta, dois problemas cardíacos, dois diabetes mellitus e dois depressão. Em relação ao uso de medicamentos, cinco o faziam somente por ordem médica e quatro dos cuidadores afirmaram não fazer uso de medicações.

Todos os cuidadores afirmaram necessitar de ajuda para o cuidado do idoso. No entanto, somente quatro conseguiam essa ajuda dos filhos, os restante não contavam com ajuda, seja de seus familiares ou cuidadores formais. Quando questionados em relação ao tempo gasto no cuidado do idoso, o tempo variou entre quatro e doze horas por dia, perfazendo, em média, oito horas diárias, o que demonstrou que os cuidadores dedicam uma boa parte do seu tempo ao cuidado, além de continuarem desempenhando suas funções 
rotineiras.

Após a síntese das histórias das experiências de cuidado, emergiram quatro categorias, discutiremos a seguir uma delas: o processo de cuidar do idoso com IRCT em DPAC no domicílio.

O cuidado é um processo humano intersubjetivo, no qual o objetivo é a proteção, engrandecimento e preservação da dignidade humana. O cuidado envolve desejo e comprometimento com o cuidar, conhecimento, ações e conseqüências do cuidado ${ }^{(16)}$.

Neste sentido, o processo de cuidar e ser cuidado são permeados pelas concepções de vida, saúde, doença e cuidado dos indivíduos, sendo influenciado pelas relações familiares e sociais. Nesta linha de pensamento, a análise das práticas de cuidado da família ao indivíduo idoso revela as regras, os procedimentos que seguem tais indivíduos, enfim suas concepções de cuidado.

Notou-se que o cuidado apresentou significados diferentes para os cuidadores deste estudo como podemos verificar nos relatos que se seguem:

... logo após o meu casamento passei por necessidades eprecisei ir morar com minha mãe e ela me recebeu de braços abertos. Agora que descobrimos a doença e que ela precisa fazer diálise é bora de retribuir... (cuidadora 6).

Cuidar da mãe, para a cuidadora 6, é uma forma de retribuir o que a idosa já fez por ela. O dever de cuidar do idoso pode ser entendido como um sentimento natural, ligado a um compromisso que foi sendo construído ao longo da convivência familiar.

... somos casados há 48 anos e faz seis anos que cuido dela. Nós temos oito filhos, mas nenbum me ajuda. Então, sou obrigado a cuidar. Um dia meu sogro disse que eu deveria deixá-la e eu respondi que não, que tenho a obrigação de cuidar, pois foi isso que prometi diante do padre... (cuidador 9).

Para o cuidador 9 o cuidado significava uma obrigação matrimonial, pois o mesmo havia feito o juramento perante Deus, por ocasião do matrimônio, em que se comprometeram a partilhar alegrias e tristezas, além de estarem juntos na saúde e na doença. Neste caso, a opção de cuidar é vista como uma obrigação que está embutida no seu papel de esposo.

O cuidar está presente no dia a dia das pessoas, é uma atividade necessária para auxiliar no desenvolvimento e crescimento de todo ser vivo, preservar objetos, resguardar e manter a vida ${ }^{(14)}$.

A atividade de cuidar de um idoso dependente é, basicamente, realizada no domicílio, no qual se desenvolve uma parte significativa da vida e, portanto, onde existe o conhecimento e a memória de fatos e de relações íntimas consideradas importantes, tanto para o cuidador quanto para quem é cuidado ${ }^{(17)}$.

Frequentemente, o cuidador que assume a atividade de cuidar do idoso dependente acumula atribuições, o que gera sobrecarga. A somatória de tarefas traz várias conseqüências para o cuidador, conforme narrativas:

... não tenho tempo para mim. Sou eu quem limpo, lavo, passo, cozinho, levo meus filhos para o serviço, faço a diálise e, também, vou trabalhar com o J. Ele trabalha como vendedor, porém como não enxerga bem, vou de motorista... é muito cansativo porque faço a diálise da manhã, levo meu filho para trabalhar, limpo a casa e lavo a roupa. Ao meio-dia faço a diálise, preparo o almoço para após a refeição sairmos para vender toldos. Às $18 \mathrm{~h}$ faço a outra diálise, preparo o jantar e passo roupa. $E$ a meia noite faço a última troca do dia... (cuidadora 8).

... a diálise está atrapalhando minha vida. Sou manicure e, às vezes, perco unha porque eu tenho que fazer a diálise da minha sogra... (cuidadora 4).

A cuidadora 8 acumulou as tarefas de cuidar da casa, dos filhos, do idoso, deixou claro como é difícil e cansativo conciliar todas as atividades. Este acúmulo de atividades leva o cuidador a um desgaste físico e mental para o qual, na maioria das vezes, nada é feito para amenizar.

Já para a cuidadora 4, a atividade de fazer a troca de bolsas de diálise trouxe conseqüências econômicas, pois a impossibilitava de realizar seu trabalho, que gerava renda para a família.

Pelos relatado, podemos inferir que o acúmulo de tarefas interfere na condição de vida dos cuidadores, sendo a sobrecarga a conseqüência mais comum. São muitas as dificuldades mencionadas pelos cuidadores, principalmente as de ordem biopsicossociais, conforme descritas nas narrativas a seguir:

... a diálise já trouxe problemas com meu marido. Ele entende e aceita que eu ajude minha mãe com a diálise, mas acha que meus irmãos deveriam ajudar... quando ele quer sair e eu não posso, por causa da diálise, ele fica bravo, fala que eu devo dar um jeito, que alguém tem que me ajudar... (cuidadora 6).

... eu não percebia que estava fazendo falta, que minhas filhas e meu esposo precisavam de mim. Eu deixei de lado minhas filhas, não dava mais atenção, não as levava a escola, não saía com elas. E meu esposo já estava cansado, eu não estava dando atenção a ele como esposa e sabe como é homem ... (cuidadora 7).

Para as cuidadoras 6 e 7, a realização da DPAC no domicílio fez com que as mesmas modificassem a rotina diária de suas famílias, o que gerou conflitos com os filhos e, principalmente, com os maridos. Ambas as cuidadoras enfrentaram problemas conjugais por assumirem a atividade de cuidar, pois deixaram de se dedicar exclusivamente à família, para dedicar-se ao idoso dependente. A atividade de cuidar exige que o cuidador dedique-se por inteiro a este processo, o que leva os mesmos a redefinir o dia-a-dia da família. 
Com o surgimento de uma doença crônica podem ocorrer perdas na esfera pessoal, social e econômica, tanto para o idoso que vivencia tal situação, quanto para a família, o que dificulta os relacionamentos sociais e o desempenho profissional. Para o cuidador, além do cansaço físico e emocional decorrente da atividade de cuidar, também ocorre a perda da liberdade, pois há a necessidade de "abrir mão" de atividades sociais para prestar o cuidado. Ser cuidador de idoso dependente no domicílio é, na maioria das vezes, negligenciar sua própria vida, pois a prioridade é cuidar do familiar doente $e^{(18-19)}$.

Apesar das perdas de ordem pessoal, social e econômica que a atividade de cuidar acarreta, os cuidadores deste estudo identificaram pontos que consideravam positivos na experiência de cuidar.

... para mim cuidar dele é como se eu estivesse cuidando do meu pai... (choro)... eu cuidava com amor, pois ele e a dona $E$. sempre nos ajudaram, cuidaram dos meus filhos... (cuidadora 3).

... é muito gratificante para mim cuidar do meu pai porque eu cuido de várias pessoas que não conheço e hoje estou cuidando do meu pai, é uma forma de retribuir o que ele já fez por mim...(cuidadora 1).

... como sou da área da saúde é um aprendizado, é uma vantagem a mais... (cuidadora 5 ).

Para as cuidadoras 1 e 3 , cuidar do idoso dependente é uma forma de retribuir o que os mesmos já fizeram por elas. Já para a cuidadora 5 , a atividade de cuidar é uma forma de aperfeiçoar seus conhecimentos.

Constatou-se também, neste estudo, que todos os cuidadores precisavam de ajuda para desempenhar o cuidado, porém a maioria não a conseguia.

... eu não tenho ajuda para fazer a diálise, principalmente da minha filha. Ela não é uma filha que ajuda, de jeito nenbum. Meu neto quando vai em casa joga as batatas da fruteira no chão e ela não faz nada, não ajuda a pegar, do jeito que ele joga fica. Imagina fazer a diálise... (cuidadora 2).

... somente eu a trago (mãe) ao hospital, porque uma irmã não dirige, a outra tem medo. Existe um certo conflito, nós não soubemos trabalhar com essa parte, não soubemos dividir as coisas... houve algumas desavenças, discussões, então ficou tudo para mim... (cuidadora 7).

... nós tivemos oito filhos, mas somente dois filhos ajudam financeiramente, pois moram longe. Os outros não vão nem visitar a mãe, não sabem o que acontece no dia a dia. Eu também sou idoso, tenho problemas de saúde, mas eles não se importam com isso. Quando preciso fazer algo, sou obrigado a deixar minha neta com a M., já houve muitas discussões, mas nada adiantou, ninguém ajuda... (cuidador 9).

Pela fala dos narradores, fica evidente a falta de ajuda para o cuidado com o idoso. É comum discussões entre os membros da família na tentativa de conseguir a ajuda de outro. O cuidador familiar, com freqüência, desempenha seu encargo sozinho, sem a ajuda de outros familiares ou de profissionais $^{(20)}$.

Cuidar do idoso em casa é uma prática que deve ser estimulada; todavia, cuidar de um indivíduo dependente durante 24 horas por dia, sem uma política de proteção para o desempenho desse papel, não é tarefa para uma única pessoa, como tem sido vivenciado por cuidadores ${ }^{(21)}$.

Pode-se constatar que as cuidadoras deste estudo manifestaram a presença do cansaço, como descrito a seguir:

... faz seis anos que eu faço a diálise em meu avô, que não tenho férias, que não posso sair a hora que en quero para dar uma volta... isso cansa, fazer a diálise em outra pessoa é cansativo, é complicado... estou cansada... (cuidadora 5).

... agora en o coloquei para me ajudar, para medir o líquido da diálise, ele pode muito bem fazer estas coisas ... eu estou cansada, preciso de ajuda, senão eu não sei o que vai acontecer comigo.... (cuidadora 8).

Ambas as cuidadoras relataram a presença de cansaço por serem responsáveis pela realização da troca de bolsa de diálise peritoneal dos idosos. Apesar do idoso possuir certa independência do hospital onde realiza o acompanhamento de seu tratamento, ele e seu cuidador necessitam realizar a troca das bolsas de diálise, até cinco vezes ao dia, o que leva a sobrecarga de trabalho e, consequentemente, ao cansaço.

Os cuidadores podem entrar em desespero, isolamento, redução do tempo livre, fadiga intensa, redução do envolvimento com a comunidade e com as atividades religiosas com a sobrecarga do cuidado. Os sintomas de fadiga são complexos, pode estar associado com a depressão e, muitas vezes, não são diagnosticados ${ }^{(9)}$.

Assim, acreditamos que ser cuidador de um idoso com IRCT em DPAC no domicílio é uma experiência única e individual. A maneira como este cuidado será realizado dependerá da relação de afeto entre seus membros e como estes entendem o processo de envelhecimento. É necessário que o enfermeiro oriente o idoso e seus cuidadores sobre a importância da DPAC e incentive-os a uma vida harmoniosa junto a sua família e amigos, dentro de suas limitações.

\section{CONSIDERAÇÕES FINAIS}

Os cuidados do idoso com IRCT em tratamento de DPAC são assumidos, na maioria das vezes, por um cuidador familiar que reside junto com o mesmo. Esse cuidador recebe, do enfermeiro responsável pelo Serviço de Diálise do hospital, treinamento para realizar a técnica de troca da bolsa de diálise, bem como orientações sobre higiene, alimentação e ingesta hídrica, cuidados com o cateter, possíveis intercorrências e complicações decorrentes da doença e do tratamento. 
A atividade de cuidar, geralmente, é de responsabilidade de uma pessoa do sexo feminino (esposa, filha ou nora), casada e, portanto, com outras responsabilidades. Assim, o cuidar do idoso dependente mais os afazeres do dia a dia, geram o acúmulo de tarefas, acarretando para o cuidador sobrecarga de trabalho, o que foi revelado neste estudo.

A atividade de cuidar é cansativa, estressante e solitária. Apesar dos treinamentos e orientações recebidas do enfermeiro, o cuidador percebe-se diante da necessidade de realizar um procedimento com determinado grau de complexidade e de extrema importância para a saúde do idoso, no qual não pode haver erros.

Considera-se que a reorganização da atenção à saúde, principalmente no que se refere ao cliente idoso é urgente.

\section{REFERÊNCIAS}

1. Silvestre JA, Kalache A, Ramos LR, Veras RP. O envelhecimento populacional brasileiro e o setor saúde. Arq Geriatr Gerontol. 1996;0(1):81-9.

2. Chaimowicz $F$. A saúde dos idosos brasileiros às vésperas do século XXI: problemas, projeções e alternativas. Rev Saúde Pública. 1997;31(2):184-200.

3. Fries JF. Measuring and monitoring success in compressing morbidity. Ann Intern Med. 2003;139(5 Pt 2):455-9.

4. Thomé FS, Gonçalves LFS, Manfro RC, Barros E, Prompt CA, Karohl C. Insuficiência renal crônica. In: Barros E, Manfro RC, Thomé FS, Gonçalves LFS. Nefrologia: rotinas, diagnóstico e tratamento. 3a ed. Porto Alegre: Artmed; 2006. p.381-404.

5. Abensur H, Castro MCM. Métodos dialíticos. In: Cruz J, Praxedes JN, Cruz HMM. Nefrologia. $2^{a}$ ed. São Paulo: Sarvier; 2006. p. 273-84.

6. Carreira L, Marcon SS. Cotidiano e trabalho: concepções de indivíduos portadores de insuficiência renal crônica e seus familiares. Rev Latinoam Enferm. 2003;11(6):82331.

7. Saraiba E, Nanches N. Diálisis peritonial. In: Andres J, Fortuny C. Cuidados de enfermería en la insuficiencia renal. Barcelona: ELA Editorial Libro del Año; 1994. p. 223-48.

8. Gonçalves LHT, Alvarez AM, Santos SMA. Os cuidadores leigos de pessoas idosas. In: Duarte YAO, Diogo MJD. Atendimento domiciliar: um enfoque gerontológico. São Paulo: Atheneu; 2005. p.101-13.

9. Schneider RA. Chronic renal failure: assessing the Fatigue Severity Scale for use among caregivers. J Clinic Nurs. 2004;13(2):219-25.

10. Given B, Sherwood PR. Family care for the older person with cancer. Semin Oncol Nurs. 2006;22(1):43-50.
São necessárias ações como educação continuada dos profissionais da saúde para que estes possam atender a essa parcela populacional. Acreditamos na importância da manutenção do idoso no próprio domicílio, cabendo à família a responsabilidade de implementar o cuidado, no caso a realização da troca de bolsa de diálise. Porém, é necessário que exista o subsídio e o suporte necessário do sistema formal para que seja realizado um cuidado de melhor qualidade.

Assim, acreditamos que o enfermeiro responsável pela DPAC deve, por meio das visitas domiciliares, identificar as necessidades que os cuidadores enfrentam durante $o$ processo de cuidar e, assim, colaborar para a assistência ao idoso e ao cuidador que necessitam de atenção.

11. Amirkhanyan AA, Wolf DA. Parent care and the stress process: findings from panel data. J Gerontol B Psychol Sci Soc Sci. 2006;61(5):S248-55.

12. Tier CG, Fontana RT, Soares NV. Refletindo sobre idosos institucionalizados. Rev Bras Enferm. 2004;57(3):332-5.

13. Meihy JCSB. Manual de história oral. São Paulo: Loyola; 1996.

14. Marques S. Cuidadores familiares de idosos: relatos de histórias [dissertação]. Ribeirão Preto: Escola de Enfermagem de Ribeirão Preto da Universidade de São Paulo; 1999.

15. Minayo MCS. O desafio do conhecimento: pesquisa qualitativa em saúde. 8a ed. São Paulo: Hucitec; 2006.

16. Watson J. Nursing: human science and human care: a theory of nursing. Norwalk: Appleton-Century Crofts; 1985.

17. Mendes PBMT. Cuidadores: heróis anônimos do cotidiano. In: Karsch UMS, organizador. Envelhecimento com dependência: revelando cuidadores. São Paulo: EDUC; 1998. p. 87-145.

18. Silva L, Bocchi SCM. A sinalização do enfermeiro entre os papéis de familiares visitantes e acompanhante de adulto e idoso. Rev Latinoam Enferm. 2005;13(2):180-7.

19. Kusumota L. História oral da insuficiência renal crônica terminal e do tratamento dialítico de idosos [dissertação]. Ribeirão Preto: Escola de Enfermagem de Ribeirão Preto da Universidade de São Paulo; 2001.

20. Neri AL. Bem estar e estresse em familiares que cuidam de idosos fragilizados e de alta dependência. In: Neri AL, organizador. Qualidade de vida e idade madura. $7^{\text {a }}$ ed. Campinas: Papirus; 2007. p. 237-85.

21. Cattani RB, Girardon-Perlini NMO. Cuidar do idoso doente no domicílio na voz de cuidadores familiares. Rev Eletrônica Enferm. 2004;6(2):254-71. 